\title{
Transformation of Nonstationary Signals into Pseudostationary Signals for the Needs of Vehicle Diagnostics
}

\author{
J. DZIURDŹ* \\ Faculty of Automotive and Construction Machinery Engineering, Warsaw University of Technology \\ Narbutta 84 02-524 Warsaw, Poland
}

\begin{abstract}
Research concerning the development of diagnostic methods of the state of the vehicle driving systems basic elements - have been carried on for many years. Modern diagnostic systems, regardless of being technically highly advanced or small degree only, concern about assessments of basic mechanical subsystems such as toothed gears or suspension elements. The information on state changes of mechanical systems can be obtained by an analysis of changes of nonlinear effects in such systems operations. Since the nonlinearity increase accompanies the system destruction, the separation of nonlinear parts of signals creates the possibility of the increase of an accuracy, reliability and application range of diagnostic methods. Technical state diagnostics for vehicles is much more difficult than for machines operating in stationary conditions. the main excitation source is an internal combustion engine, in which a rotational speed changes from an idle running (approximately $800 \mathrm{rpm}$ ) to a maximum rotational speed (e.g. above $5000 \mathrm{rpm}$ ). Thus, an application of classic analytical methods is not possible. the only solution is the transformation of the analysed signals into the "pseudostationary" ones, by taking into account changes in the engine rotational speed. One of the methods applied now a days is resampling (adaptation change of a signal time resolution in a digital form) performed on the basis of information concerning changes in an engine rotational speed. the signal from the sensor of the engine crankshaft location was used for the above application.
\end{abstract}

PACS numbers: $46.40 .-\mathrm{f}, 46.80 .+\mathrm{j}$

\section{Introduction}

Investigations of physical phenomena, which occur in mechanical systems are usually based on the analysis of the recorded signals of physical quantities, e.g.: vibration accelerations or acoustic pressure changes near the object under testing. Signals can be divided — in theory into the determined and the random ones [1], while in practice - the recorded signals consist of the parts determined with a higher or lower random disturbance. Analysis of these effects is relatively simple at constant excitation parameters (steady state working conditions). However, in actual systems, this condition is not always satisfied, e.g. when the rotational speed of the drive being the basic kinematic excitation of the system changes.

Basic reasons of irregular operations of mechanical driving systems are load changes and related to them changes in the rotational speed, which depend on the characteristics of system elements (including a driving engine) and changes of the rotational speed related to the specificity of system operations (e.g. uneven operation of system elements). There are also certain other reasons including the most essential one - which is the influence of the control performed by the operator of the

\footnotetext{
* corresponding author; e-mail: jdz@simr.pw.edu.pl
}

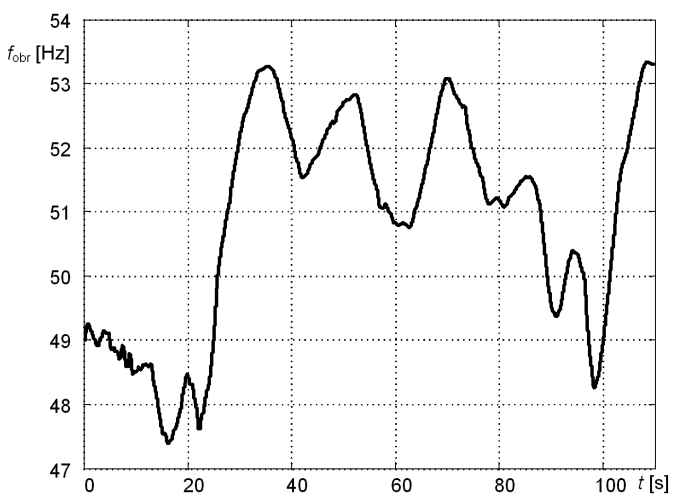

Fig. 1. The change of the rotational speed of the driving engine during testing the constant speed drive.

device.

The rotational speed of the engine, in cars without the automatic system of maintaining the constant driving speed, depends on the actual load (changing over time) and on the position of the accelerator pedal controlled by the driver. The change of the rotational speed of the driving engine $f_{\text {rot }}$ during testing the constant speed drive is shown, as an example, in Fig. 1.

The accurate analysis of a mechanical system performance, at such rotational speed changes, is only pos- 
sible when applying the nonstationary signal analysis (e.g. wavelet transform, Wigner-Ville transform, etc.). The analysis is very difficult — possible in a limited range only and impossible at larger changes - when the Fourier Transform is applied. This is also reflected in the recorded signals of physical quantities.

One of the possibilities improving the situation is such transformation of the signal (obtained from the signal measurement) that it corresponds to the signal obtained for the constant rotational speed, which requires the signal resampling [2].

\section{Determination of the changes of the engine rotational speed}

\subsection{Analysis of the signal obtained from the inductive sensor}

Information concerning the rotational speed changes is useful at testing rotations of mechanical systems. This information is necessary if one wants to perform the signal resampling. Tachometers, optoelectronic sensors as well as encoders enabling the accurate recording of the rotational speed are used for this purpose.
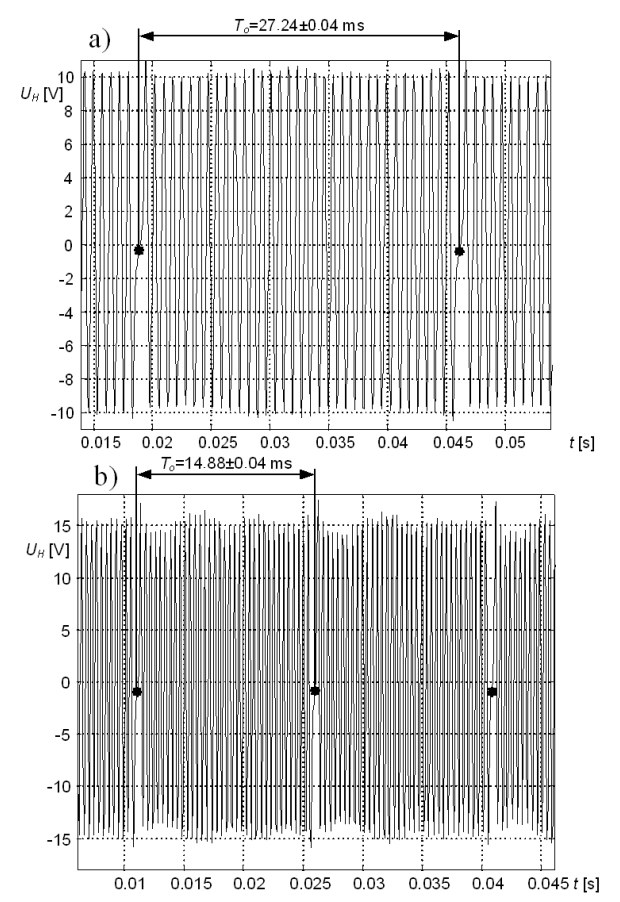

Fig. 2. Recorded voltage changes from the inductive sensor with the marked duration time of one rotation of the shaft: (a) $n_{\text {rot }}=2200 \mathrm{rpm}$, (b) $n_{\text {rot }}=4030 \mathrm{rpm}$.

It is often impossible to apply this type of sensors in driving systems of vehicles, without any interference with their constructional elements. On the other side, the modern internal combustion engines are equipped with electronic control systems which utilise information on an angular position of a crank shaft from Inductive sensor (or Hall's sensor [3]). The sensor external voltage

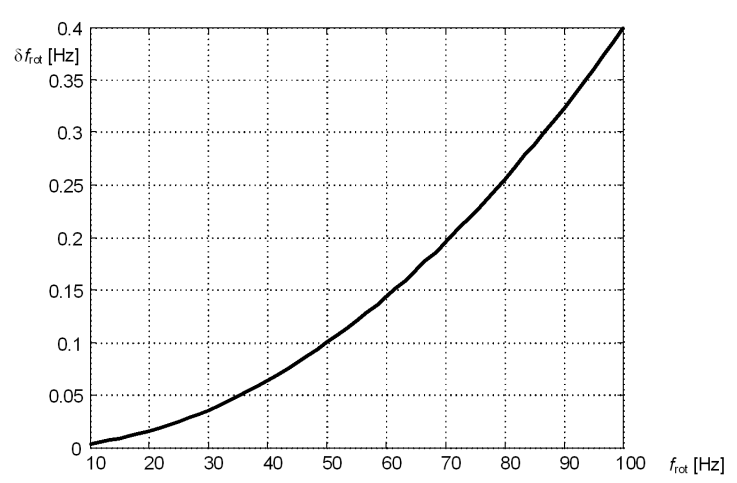

$\begin{array}{lllllllllll}600 & 1200 & 1800 & 2400 & 3000 & 3600 & 4200 & 4800 & 5400 & 6000 & n_{\text {rot }}[\mathrm{rpm}]\end{array}$

Fig. 3. Absolute error in determination of the rotation frequency $\delta f_{\text {rot }}$ for the sampling frequency $f_{s}=25 \mathrm{kHz}$.

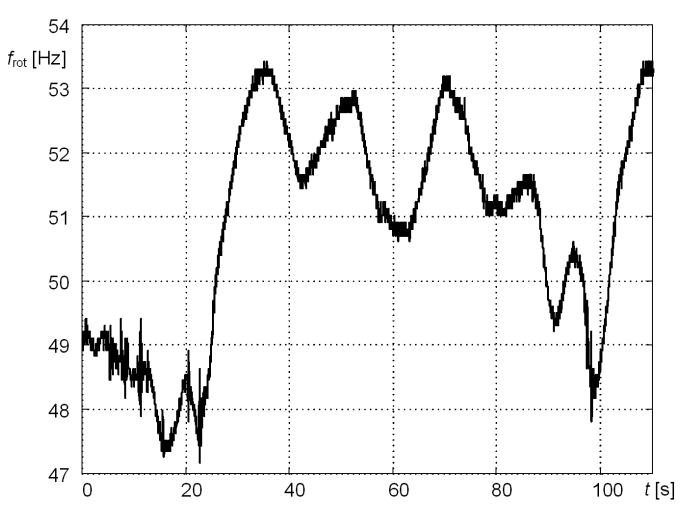

Fig. 4. The engine rotational speed change obtained from the analysis of the recorded voltage of the inductive sensor.

depends on the rotational speed and on the distance of the rotating ferromagnetic elements (most often being in a form of a plate with cut-outs or splines) from the sensor (mounted to the frame). The proper distribution of magnetic elements allows to determine explicitly the successive shaft rotations. Examples of voltage $U_{\mathrm{H}}$ changes from the inductive sensor are presented in Fig. 2 - for two rotational speeds.

Times of duration of the individual rotations can be determined on the basis of signals that are presented in Fig. 2. Unfortunately, the accuracy of such determination is limited by errors related to the signal sampling. The error in determination of the point location in the recorded signal depends on the sampling frequency $f_{s}$ and equals $\pm 0.02 \mathrm{~ms}$ for $25 \mathrm{kHz}$. The absolute error in the determination of the duration time of one rotation $\delta T$ — caused by the sampling — equals $\pm 0.04 \mathrm{~ms}$, while the relative error increases with the angular speed increase (Fig. 3).

The error related to the signal sampling influences significantly changes the determination of the rotational speed of the engine. Practically, the time-history that is obtained in such a way, is unusable in the signal resam- 
pling process (Fig. 4). Thus, how to obtain the signal similar, for instance, to the one presented in Fig. 1?

\subsection{Simulation analysis}

The solution of the problem can be presented by performing simulation examinations. The excitation signal was modelled in the form:

$$
x(t)=A \cos \left(2 \pi f_{\text {obr }} t+\delta f_{\text {obr }} \cos \left(2 \pi f_{\delta} t\right)\right)
$$

where $A$ - signal amplitude (equivalent of the voltage of the inductive sensor), $f_{\text {rot }}$ - average value of the rotational speed, $\delta f_{\text {rot }}$ - change of the rotational speed, $f_{\delta}$ - frequency of the rotational speed changes, $t$ - time.

Assuming the sampling frequency $f_{s}$ to be equal $25 \mathrm{kHz}$, the time-history of the rotational speed of the simulated signal was determined (Fig. 5a). Errors in the reproduction of the rotational speed are clearly seen. There are treated as broadband random disturbances of the signal. When the obtained signal is filtered in the band corresponding to the main components, the time-history of the rotational speed is obtained corresponding to the simulated values (Fig. 5b). This confirms the effectiveness of the signal filtration in order to remove errors caused by the sampling. Influence of filtration can be assessed by comparing spectra from Figs. $5 \mathrm{c}$ and $\mathrm{d}$.

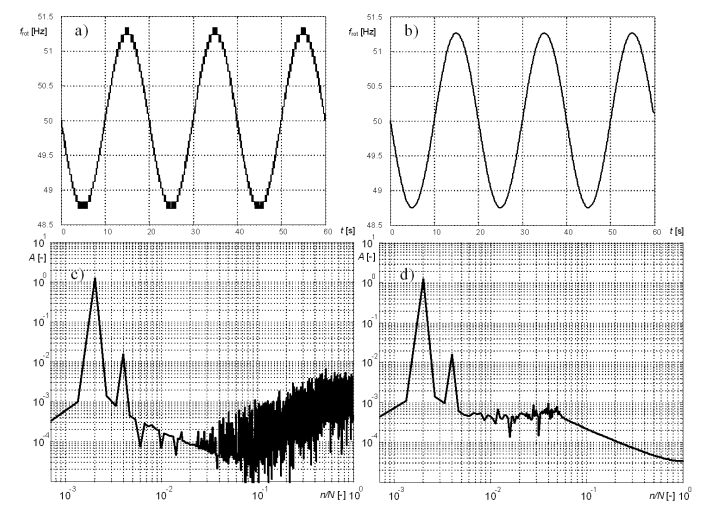

Fig. 5. Simulation of determining the rotational speed: (a) reproduced change of the rotational speed with errors caused by the sampling, (b) reproduced change of the rotational speed after filtration, (c) spectrum of the reproduced change of the rotational speed with errors caused by the sampling, (d) spectrum of the reproduced change of the rotational speed after filtration.

\section{Signal resampling}

\subsection{Method description}

The problem of the signals resampling concerns their digital form, where - instead of the continuous functions - sets of the successive amplitudes of the physical quantities are obtained by recording. Those amplitudes are measured in the time-moments being distant from each other by a constant value $\Delta t$, equal to the reciprocal of the sampling frequency $f_{s}$. Thus, the resampling means the reproduction of amplitudes in different time-moments and the applied methods can be divided into uniform and not uniform (dynamic) over time. In case of mechanical systems, there is a need of applying the dynamic resampling, which takes into consideration the change of the engine rotational speed. The needed data can be obtained by means of the procedure of using the inductive sensor. The dependence between time and successive rotations of the engine shaft can be estimated on the basis of information acquired from this sensor.

Assuming a certain rotational speed $f_{\text {rot }}$ (the most convenient is the value close to the average one in the analysed time interval) it is possible to determine the successive values of angle $\varphi_{i}$, corresponding to the engine uniform operation. The constant increase of the angle of rotation of the shaft - in time $\Delta \varphi$ - orresponds to the constant rotational speed:

$$
\Delta \varphi=2 \pi \frac{f_{\text {rot }}}{f_{s}},
$$

where: $f_{\text {rot }}$ - assumed value of the rotational speed, $f_{s}$ - frequency of the signal sampling.

The determined values of the angle of rotation of the shaft $\varphi_{i}$ can be used for the determination of the time needed $t_{i}\left(\varphi_{i}\right)$ in the actual signal. In order to achieve this purpose, the functional dependence between the time and the angle of rotation of the shaft should be determined. Utilising the previously determined time $t_{j}$ values for the successive rotations and assuming that this function should be continuous and differentiable, the interpolation is performed (e.g. by spline functions [4]). The algorithm of the selected time moment $t_{i}\left(\varphi_{i}\right)$ is presented in Fig. 6 .

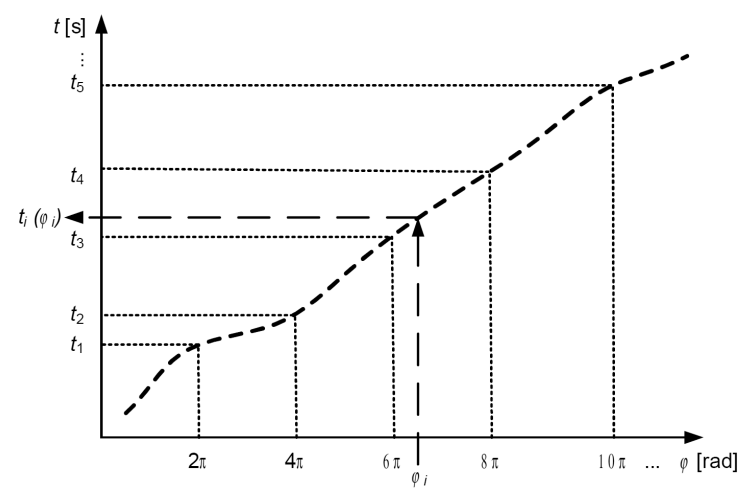

Fig. 6. Change of time as a function of the angle of rotation of the shaft and the example of the time determination $t_{i}\left(\varphi_{i}\right)$.

Similar problem concerns the reproduction of signal amplitudes for $t_{i}$ values. This can be done in a similar fashion by means of the approximation of the recorded signal time-history. The example of the resampled signal is presented in Fig. 7. 


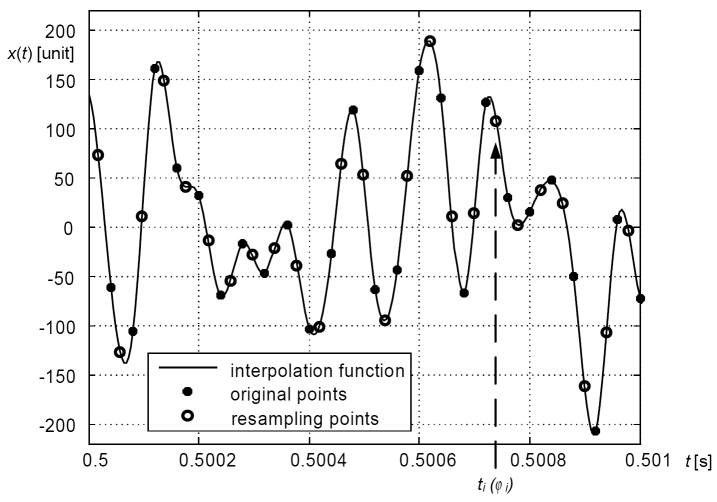

Fig. 7. Example of the signal resampling.

\subsection{Test of the operation}

Test of the operation of the presented method was carried out by using the simulation signal described by Eq. (1). The times of the successive rotations of the shaft were calculated on its basis. The same signal, but with the noise added as a random disturbance, was used as the signal for the resampling procedure. The resampling was performed for the assumed rotational frequency of the shaft $f_{\text {rot }}$ being equal $50 \mathrm{~Hz}$. Instantaneous spectra (for $\Delta f=1 / 60 \mathrm{~Hz}$ ) as well as averaged spectra (for 100 averages, $\Delta f=0.763 \mathrm{~Hz}$ ) of signals before and after the modification are shown in Fig. 8.

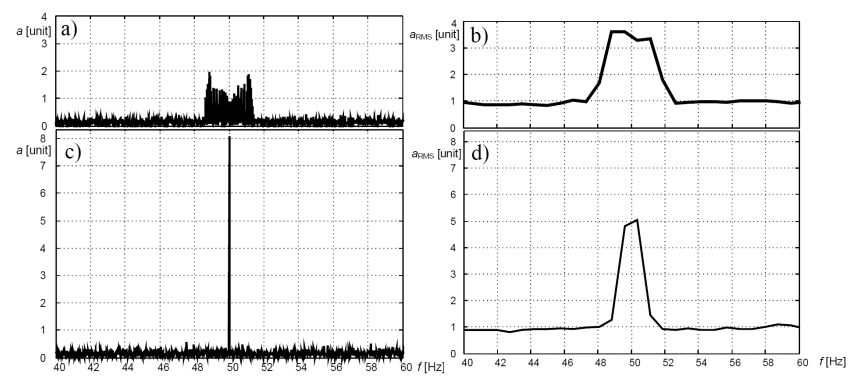

Fig. 8. The simulation results: (a) instantaneous spectra of the signal before the modification, (b) instantaneous spectra of the signal after the modification, (c) average spectra of the signal before the modification,

(d) average spectra of the signal after the modification.

The frequency resolution of the Fourier transform is inversely proportional to the duration time of the run being analysed [5]. The spectrum of the modulated signal is obtained for long time analysis (Fig. 8a) while for short averaged runs (Fig. 8c) the sum of spectra of various instantaneous frequencies of the harmonic component is obtained. That is why for the signal which was not resampled the broader band of the essential components in which it is not possible to separate the characteristic harmonic component $f_{\text {rot }}$ - is obtained.

The results of analysis presented hereby confirm effectiveness of the proposed method. The representation of the basic signal frequency obtained after the modification was quite satisfactory.

\section{Analysis carried out for the actual driving system}

The results of analysis of the signals recorded when going by a passenger car are given as the example of the method effectiveness in testing actual objects. The signal from the inductive sensor was used for the estimation of dependence between time and the successive rotations of the engine shaft. The signal of vibration acceleration of the cylinder head was resampled. A time segment of 60 -second duration was selected from the recording shown in Fig. 4. The reproduced change of the rotational frequency of the engine is presented in Fig. 9.
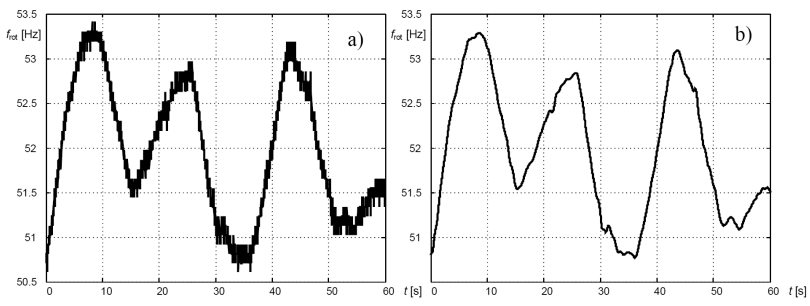

Fig. 9. Change of the rotational frequency of the engine for $60 \mathrm{~s}$ run: (a) before filtering the disturbances, (b) after filtering the disturbances.

Spectra of the vibration signal, before and after resampling, are illustrated in Fig. 10.

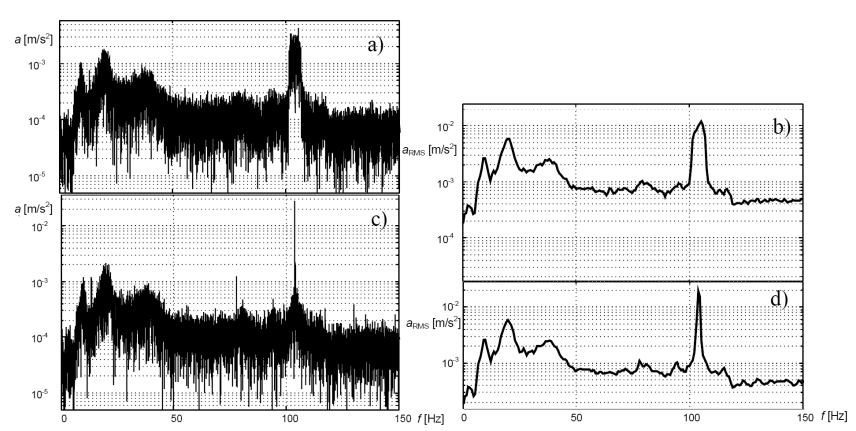

Fig. 10. Spectra of acceleration of the cylinder head vibrations: (a) instantaneous before the resampling, (b) averaged before the resampling, (c) instantaneous after the resampling, (d) averaged after the resampling.

In a similar fashion as in the case of simulation the instantaneous spectra are presented with the frequency resolution $\Delta f=1 / 60 \mathrm{~Hz}$, while the averaged spectra with the resolution $\Delta f=0.763 \mathrm{~Hz}$ (100 averages).

It is not possible to separate - in the obtained spectra - the component related to the rotational frequency of the engine, being app. $52 \mathrm{~Hz}$. The most essential component is related to the ignition frequency $f_{\text {ign }}$ equal app. $104 \mathrm{~Hz}$ (for the four-cylinder four-stroke engine this 
frequency equals the double value of the rotational frequency).

The comparison of the spectra presented in Fig. 10 allows to state, that the proposed method is equally effective for the simulated signals and for the ones recorded in actual objects.

\section{Conclusions}

It should be emphasised the resampled signal is not the equivalent of the signal recorded in stationary conditions. Changes related to the system kinematics are taken into account at the signal modification while the dynamic phenomena are not considered. A good example of this effect can be the resampling of the signal recorded in the system operating with the varying rotational speed during the passage of the mechanical resonance.

The system with the inductive sensor applied in vehicles, regardless of its low accuracy, seems to be an adequate information source related to changes of the engine rotational speed. Applying the filtration - in order to decrease the error influences - significantly improves the representation accuracy.

The algorithm proposed in the present paper constitutes the alternative for methods of analysis of nonsta- tionary signals (e.g. time-frequency). It enables comparing the results of road measurements with the measurements performed in stationary conditions (at the measuring stands) or during tests on the chassis test bed.

The presented results of the simulation investigations as well as the analysis of signals from the actual objects confirm the effectiveness of the proposed method. This method allows the application of techniques of signal transformation, which require a large number of averages (e.g. coherence function).

\section{References}

[1] J.S. Bendat, A.G. Piersol, Random data: Analysis and measurement procedures, 2nd ed., John Wiley, New York 1986

[2] F. Bonnardot, M. ElBadaoui, R.B. Randall, J. Daniere, F. Guillet, Mechanical Systems and Signal Processing 19, 766 (2005).

[3] E. Ramsden, Hall-Effect Sensors: Theory and Application, 2nd Ed., Newnes, an imprint of Elsevier.

[4] C. de Boor, Spline Toolbox for use with MATLAB User's Guide, Version 3.1.1, The Mathworks, Natick (USA) 2002

[5] R.B. Randall, Frequency Analysis, Brüel\&Kjær, Denmark 1987. 